\title{
artigo
}

Gomes Silva, M.; Estrela, F.M.; Peixoto, T.M.; Moreira, R.M.O.; Morais, A.C.; Couto, P.L.S.; Portela, P.L.S.

Violência contra a mulher em Feira De Santana-BA: Encaminhamentos e articulação em rede

\section{Violência contra a mulher em Feira De Santana-BA: Encaminhamentos e articulação em rede}

\author{
Violence against women at the Feira De Santana-BA: Networks and joints \\ Violencia contra mujeres en la Feira De Santana-BA: Redes y juntas
}

\begin{abstract}
RESUMO
Objetivo: descrever a percepção da equipe de saúde no que tange à atenção às mulheres em situação de violência a resolutividade das demandas e encaminhamentos em rede. Método: estudo de caráter exploratório-descritivo, de abordagem qualitativa com 18 participantes entre profissionais de saúde e gestores. A coleta foi realizada através de entrevistas diretamente in loco junto aos participantes que devem gerir e executar as ações de prevenção na Atenção Básica do município de Feira de Santana-BA. $O$ estudo respeitou os preceitos éticos do Conselho Nacional de Saúde. Resultado: 0 estudo revelou que os profissionais colocam que não sabem lidar diante da situação de violência, não conhecem os fluxos para encaminhamento e apontam para a necessidade de maior divulgação desses a fim de garantir à mulher o atendimento às suas necessidades. Conclusão: É de fundamental importância sugerir disciplinas que contemplem, em seus currículos e programas de educação continuada, a formação e treinamento dos aspectos relacionados com a violência.
\end{abstract}

DESCRITORES: Violência; Mulheres; Pessoal de Saúde; Estratégia Saúde da Família.

\section{ABSTRACT}

Objective: to describe the perception of the health team regarding the attention to women in situations of violence and the resolution of demands and referrals in the network. Method: an exploratory-descriptive study with a qualitative approach with 18 subjects, including health professionals and managers 18 (eighteen) subjects. The collection was carried out through interviews directly in loco with the actors who must manage and execute the preventive actions in Primary Care in the municipality of Feira de Santana-BA. The study respected the ethical precepts of the National Health Council. Result: The study revealed that professionals say they do not know how to deal with the situation of violence, do not know the flows for referral and point to the need for greater dissemination of these in order to guarantee women to meet their needs. Conclusion: It is of fundamental importance to suggest subjects that include, in their curricula and continuing education programs, the formation and training of aspects related to violence.

DESCRIPTORS: Violence; Women; Health Personnel; Family Health Strategy.

\section{RESUMEN}

Objetivo: describir la percepción del equipo de salud sobre la atención a las mujeres en situaciones de violencia y la resolución de demandas y derivaciones en la red. Método: estudio exploratorio-descriptivo con abordaje cualitativo con 18 sujetos, incluyendo profesionales de la salud y gestores 18 (dieciocho) sujetos. La recolección se realizó a través de entrevistas directamente in loco a los actores que deben gestionar y ejecutar las acciones preventivas en Atención Primaria en el municipio de Feira de Santana-BA. El estudio respetó los preceptos éticos del Consejo Nacional de Salud. Resultado: El estudio reveló que los profesionales dicen no saber cómo lidiar con la situación de violencia, desconocen los flujos de derivación y señalan la necesidad de una mayor difusión de estos. para garantizar que las mujeres satisfagan sus necesidades. Conclusión: Es de fundamental importancia sugerir materias que incluyan, en sus currículos y programas de educación continua, la formación y capacitación de aspectos relacionados con la violencia.

DESCRIPTORES: Violencia; Mujer; Personal de Salud; Estrategia de Salud de La Familia

RECEBIDO EM: 29/01/2021 APROVADO EM: 15/02/2021

\section{Marcia Gomes Silva}

Enfermeira. Mestre em Gestão de Políticas Públicas. Docente da Universidade Estadual de Feira de Santana.

ORCID: 0000-0001-5571-0649 


\section{Fernanda Matheus Estrela}

Enfermeira. Doutora em Enfermagem e Saúde. Docente da Universidade Estadual de Feira de Santana.

ORCID: 0000-0001-7501-6187

\section{Thais Moreira Peixoto}

Enfermeira. Mestre em Enfermagem e Saúde. Docente da Universidade Estadual de Feira de Santana, Feira de Santana, Bahia Brasil. ORCID: 0000-0001-5395-0905

\section{Tânia Maria de Oliveira Moreira}

Enfermeira. Docente da Universidade Estadual de Feira de Santana.

ORCID: 0000-0002-4541-6750

\section{Ariane Cedraz Morais}

Enfermeira. Mestre em Enfermagem. Docente da Universidade Estadual de Feira de Santana, Feira de Santana, Bahia Brasil. ORCID: 0000-0001-9445-4596

\section{Pablo Luiz Santos Couto}

Enfermeiro. Mestre em em Enfermagem e Saúde Docente da Universidadedo Estado da Bahia. ORCID: 0000-0002-2692-9243

\section{Pollyana Pereira Portela}

Enfermeira. Mestre em Enfermagem. Docente da Universidade Estadual de Feira de Santana. ORCID: 0000-0002-6840-4533

\section{INTRODUÇÃO}

A violência tem presença marcante na construção histórica da humanidade em seus vários momentos. Trata-se de um processo complexo, multicausal, pautada na construção social de gênero, sendo necessário a compreensão e conhecimento pelos profissionais de saúde para os encaminhamentos em rede necessários.

Segundo dados do Fórum Brasileiro de Segurança Pública, apenas nos meses de março a maio de 2020, 189 mulheres foram assassinadas no Brasil, o que representa um aumento, embora pequeno, considerável de 2,2\% em relação ao ano anterior ${ }^{1}$. Com base nesses dados do Sistema de Informação sobre Mortalidade (SIM) não é possível, contudo, identificar que parcela corresponde às vítimas de feminicídio (um termo de crime de ódio baseado no gênero, amplamente definido como o assassinato de mulheres, mas as definições variam dependendo do contexto cultural), uma vez que a base de dados não fornece essa informação. Ainda que a taxa de homicídio de mulheres tenha crescido 7,5\% entre 2005 e 2015, quando se analisam os anos mais recentes, verifica-se uma melhora gradual, tendo este indicador diminuído $2,8 \%$, entre 2010 e 2015 , e sofrido uma que- da de 5,3\% apenas no último ano da série 2 . Ao analisar o ano de 2019 e comparado com o ano de 2018, os casos de feminicídio tiveram um aumento de $7,3 \%$, o que representa a morte de uma a cada 7 horas ${ }^{3}$.

Os dados indicam ainda que, além da taxa de mortalidade de mulheres negras ter aumentado, cresceu também a proporção de mulheres negras entre o total de mulheres vítimas de mortes por agressão, passando de $54,8 \%$ em 2005 para $65,3 \%$ em 2015. Assim, 65,3\% das mulheres assassinadas no Brasil no último ano eram negras, na evidência de que a combinação entre desigualdade de gênero e racismo é extremamente perversa e configura variável fundamental para compreendermos a violência letal contra a mulher no país².

Segundo o Relatório Mundial da Organização Mundial da Saúde sobre Violência, publicado em 2002, há um visível custo humano e elevado custo à rede de saúde pública de saúde, relativo às internaçôes e ao atendimento físico e psicológico; além de gerar repercussões no mercado de trabalho, em razão dos prejuízos ao desempenho profissional da vítima. Ao tratar a violência como um problema de saúde, o setor cria uma nova pauta interna que é, ao mesmo tempo, rica, necessária e inquietante, e pas- sa a reconhecer que esse fenômeno não é uma doença do agressor, nem uma doença da vítima, mas um sério problema social que causa agravos à saúdét

Bandeira e Almeida apontam para a invisibilidade das mulheres e da violência no Sistema Único de Saúde (SUS), situação que se relaciona às condições históricas e políticas. No Brasil, os serviços mais procurados pelas mulheres em situação de violência são o da polícia/delegacia e quando apresentam demandas de saúde, buscam as unidades de pronto atendimento (UPAS). Nesse sentido, a integralização da rede acaba por se dar apenas nesses dois serviços, buscando a resolução de crimes quando nos serviços jurídicos/policiais e a medicalização quando na unidade de saúde ${ }^{5-6}$.

O conhecimento da equipe de saúde sobre à violência e os encaminhamentos em rede poderá possibilitar o planejamento de intervenções mais adequadas às necessidades dos grupos populacionais em situação de maior vulnerabilidade. Assim sendo, partindo da ideia que a atenção primária é porta de entrada dos serviços de saúde, tendo unidades com o potencial de acolher, atender e encaminhar as mulheres, surge como problema desta pesquisa de mestrado: Qual a percepção da equipe de saúde 
no que tange à atenção às mulheres em situação de violência a resolutividade das demandas e encaminhamentos em rede?

O objetivo geral da pesquisa foi descrever a percepção da equipe de saúde no que tange à atenção às mulheres em situação de violência a resolutividade das demandas e encaminhamentos em rede.

Este estudo possui relevância de cunho acadêmico, social e profissional, haja vista que promove reflexões sobre políticas públicas de gênero. Um aspecto significativo é a possibilidade de contribuir com a comunidade acadêmica ao promover discussões acerca da temática estudada, uma vez que apresenta os dados de um estudo de caso local, refletindo a realidade vivenciada pelos profissionais da atenção primária do município de Feira de Santana-BA, local que possui uma rede de atenção à violência contra a mulher. No que se refere à relevância social, este trabalho poderá contribuir para identificar e divulgar quais ações preventivas são prestadas à comunidade que busca atendimento nas unidades primárias de saúde e, assim, fortalecer a rede de atenção.

\section{METODO}

O estudo foi de caráter exploratório e descritivo que permitem ao investigador aumentar sua experiência em torno de determinado problema, assim como, buscar a fundamentar uma discussão na área de políticas públicas, voltada para a Violência contra a Mulher nas ações de prevenção das unidades da APS ${ }^{7}$.

Utilizou-se metodologia qualitativa com realização de entrevistas semiestruturadas, que, segundo Minayo8, caracteriza-se por responder questões muito particulares, ocupando-se de uma realidade que não pode ou não deveria ser quantificada, trabalhando, por exemplo, com o universo dos significados, dos motivos, das aspirações, das crenças, dos valores e das atitudes.

Para atingir o objetivo do estudo, esta pesquisa qualitativa traçou um percurso metodológico buscando conjugar algumas etapas e técnicas investigativas pertinentes, a saber: levantamento bibliográfico, pesquisa documental e entrevistas semiestruturadas com 18 (dezoito) participantes diretamente envolvidos com o objeto em questão: 01 (um) médico, 05 (cinco) enfermeiros, 03 (três) técnicos de enfermagem, 07 (sete) agentes comunitários, 02 (dois) gestores.

A pesquisa de campo foi realizada entre 17/09/2018 a 09/10/2018, buscando informações diretamente in loco junto aos participantes que devem gerir e executar as ações de prevenção na Atenção Básica do município de Feira de Santana-BA. O critério utilizado para escolha do recorte territorial onde foi realizada a pesquisa foi o nível de violência geral no município, e os níveis em seus territórios, conforme dados da Secretária de Segurança Pública ${ }^{9}$. Foram selecionadas duas Unidades de Saúde da Família (USF) em bairros mais violentos e duas Unidades Básicas de saúde (UBS), em bairros com menores índices de violência. Nos dois bairros mais violentos (Conceição e Campo Limpo) pesquisou-se na USF Videiras e USF Campo Limpo IV; e nos dois bairros menos violentos (Serraria Brasil e Jardim Acácia) as UBS Serraria Brasil e Dispensário Santana foram utilizadas, para fins de comparação nas unidades de Atenção básica (AB). A escolha por dois territórios com maiores e menores índice de violência se deu no sentido de compreender de há diferenciação nos tipos de ações de prevenção realizadas e possível incidência destas em termos de efetividade na diminuição da violência contra a mulher.

Em relação à abordagem das entrevistas, inicialmente os participantes foram informados sobre o tema e o objetivo da pesquisa, e aqueles que se dispuseram a participar, ou seja, uma amostra aleatória da equipe multidisciplinar nas unidades supra citadas, assinaram um termo de consentimento livre e informado, que assegurou aos participantes a confidencialidade das informações fornecidas, sendo estas utilizadas exclusivamente para fins deste estudo, aprovado pelo Comitê de Ética em Pesquisa da Universidade Federal do Recôncavo da Bahia (n. 3.039.438) respeitando a Resolução $n^{\circ}$ 466/2012 do Conselho Nacional de Saúde - Ministério da Saúde.

Em relação ao método de análise de dados, realizou-se a análise das narrativas coletadas nas entrevistas, sendo associada aos referenciais de autores discutidos na revisão de literatura deste estudo.

\section{RESULTADOS}

As falas revelam que apesar de existir locais para encaminhamento das mulheres em vivência de violência, é uma rede desarticulada que precisa de maiores capacitações para que todos consigam fazer os encaminhamentos da melhor forma e que nas unidades de demanda aberta a falta de vínculo dificulta dos atendimentos e reconhecimento do agravo. A seguir a fala dos profissionais que abordam esses encaminhamentos:

Existe uma rede de atenção a mulher em vivência de violência, entretanto ela não é articulada. Essa rede é composta por serviços da área da saúde, setores jurídicos, policiais e de assistência social, a exemplo do Centro de Referência da Mulher, Delegacia Especializada no Atendimento à Mulher, CRAS, CREAS e serviços de saúde como policlínicas, UPAS e hospitais (P1)

Eu tento dialogar com a mulher no momento da consulta. Este momento é fundamental para que ocorra o acolhimento e encaminhamento diretamente para delegacia comum. Via de regra eu cuido das lesões visiveis e encaminho para dar queixa. Desconheço os fluxos para outros encaminhamentos (P2).

Por atender aqui na UBS que é demanda espontânea, não existe muito vínculo, os atendimentos são rápidos ( $P 3$ ).

Aqui na USF, incluimos enquanto atividades de prevenção à violência contra a mulher: rodas de conversas, oficinas, dinâmica de grupo, palestras e visitas domiciliares (P4). As unidades que possuem uma maior incidência de casos violentos, incluimos rodas de conversa sobre a temática (P5).

Nos encontros voltados para dis- 
cussão da temática da violência, as mulheres colocam sobre seus anseios, medos e situações particulares. É enriquecedor, elas inclusive sugerem temáticas para os próximos encontros (P6).

Eu cuido apenas das feridas e encaminho as demandas psicológicas para unidades com psicólogos, nos NASF (P7).

Eu desconheço para onde encaminhar as mulheres vitimas de violência, o município não divulga tais fluxos (P8).

Na verdade, atribuo a falta de conhecimento sobre os fluxos a falta de capacitação e porque na faculdade não tive uma disciplina que abordasse tal temática (P9).

Eu não entendo muito os fluxos de encaminhamentos, então passo para a colega resolver (P10).

Não existe um feedback quando encaminhamos os casos para a rede de atenção. A contra referência nunca vem preenchida. Sabemos apenas do relato da mulher (P11).

$A$ rede de atenção a mulher em vivência de violência não é articulada. Então quando eu preciso fazer algum encaminhamento, eu mesma ligo para tentar agendar algo (P12). Percebo que a consulta a mulher tem medo de se expor e dizer que foi violentada, principalmente aqui na UBS que não possui vínculos (P13). Aqui recebo muitos casos, mas só percebo ser violência quando a lesão é visivel (P14).

A Secretaria podia agendar capacitaçôes sobre os locais de encaminhamento e deixar a rede mais articulada (P15).

Nunca preenchi uma ficha de referência, porque os colegas não dão retorno com a contra referência, então faço o que posso voltado para os cuidados (P16).

Eu confesso que quando tenho um caso de mulher em vivência de violência, passo para a coordenação resolver (P17).
Nos que trabalhamos em PSF estamos em posição estratégica para resolver conflitos familiares e saber de casos de violência (P18).

\section{DISCUSSÃO}

\section{Encaminhamentos e articulação da rede}

Os entrevistados referem que os serviços especializados que compõem a rede de apoio e atenção às vítimas de violência contra a mulher são existentes no município conforme já mencionado. Essa rede é constituída por serviços da área da saúde, setores jurídicos, policiais e de assistência social, a exemplo do Centro de Referência da Mulher, Delegacia Especializada no Atendimento à Mulher (DEAM), Centro de Referência da Assistência Social é (CRAS), Centro de Referência Especializado de Assistência Social (CREAS) e serviços de saúde como policlínicas, UPAS e hospitais. Apesar de os profissionais afirmarem conhecer a existência de determinados serviços que atendem às vítimas de violência, relataram não conhecer a existência de uma rede intersetorial para atenção às vítimas. Observa-se que a atitude dos profissionais em orientar a busca por serviços fora da área da saúde ocorre de maneira informal, descontextualizada e não constitui rotina institucional a partir da articulação do setor saúde com os outros segmentos.

Conhecer os diversos serviços que compõem a rede de atenção e prevenção da violência é um passo importante para a articulação intersetorial. No entanto, é preciso modificar o processo de trabalho dos profissionais, pois evidenciaram dificuldades destes em funcionar, pensar e agir em rede. As atitudes profissionais ainda estão demarcadas por trabalhos isolados e desarticulados.

Ao indagar o profissional médico sobre seus encaminhamentos este colocou, de forma bem tranquila, que tenta dialogar com a vítima na consulta quando o problema tem visibilidade ou nos casos das que se sentem à vontade para falar sobre seus medos antes que a violência tenha se configurado, pensando nas questões de prevenção. Ressalta que, em todos os casos, mesmo quando a paciente tenta omitir, fica nítido no comportamento das vítimas, na forma de se posicionar diante o profissional. Acrescenta ainda na fala que na $\mathrm{AB}$ no momento da consulta a função é acolhimento e encaminhamento diretamente para delegacia comum. Ao questionar se busca a DEAM, o mesmo conclui que não, na verdade coloca que fica tão indignado que orienta a paciente a buscar qualquer delegacia.

Assim este profissional não diferencia a polícia comum para uma delegacia especializada, em que o tratamento será diferenciado, e desconhece a rede de enfrentamento do município. Logo, leva a entender a desarticulação que existe na rede, tendo em vista o encaminhamento inadequado que o médico afira dar.

Rodríguez-Bolaños, Márquez-Serrano e Kageyama-Escobar ${ }^{10}$ destacam outras barreiras que impedem a identificação e encaminhamento dos casos de violência. Para esses autores, os médicos temem por sua segurança pessoal, não querem se envolver com questões judiciais, desconhecem locais para encaminhar as mulheres e não acreditam que o serviço de saúde seja um local adequado para tratar casos de violência. Além disso, os profissionais tendem a desconfiar que as mulheres estejam realmente falando a verdade e acreditam que suas atitudes podem não contribuir para solucionar o problema. Existem também barreiras institucionais, considerando que a instituição não oferece suporte ao atendimento, nem espaços privados para realizar a consulta, sem contar a falta de capacitação profissional. Destaca-se também que não estão sendo completamente cumpridas as normas de atenção estabelecidas, que contemplam identificação, valorização do risco, fortalecimento, orientação, referência e registro.

No Sistema Único de Saúde (SUS) as interações médico-paciente também se mostraram úteis para a compreensão do fenômeno investigado. A percepção dos médicos sobre a violência perpetrada por parceiro 
íntimo é influenciada pela condição social da mulher, que é fortemente desqualificada. Principalmente nas UBS em bairros mais violentos, os atendimentos são mais rápidos por serem consultas com especialista.

Os profissionais entrevistados afirmam que em as unidades de atenção básica realizam periodicamente as seguintes atividades de prevenção à violência contra a mulher: rodas de conversas, oficinas, dinâmica de grupo, palestras, além dos fortalecimentos dos Agentes comunitários de Saúde (ACS) nas ações realizadas nas visitas domiciliares.

Segundo as enfermeiras das USF e UBS com maior número de casos de violência, quinzenalmente realizam-se rodas de conversas, dinâmica de grupo com apoio de instituições de ensino que realizam prática do curso de enfermagem, momento em que ocorre troca de informação com objetivo de provocar a reflexão do grupo provocar discussões para se ter consciência do problema e saber como enfrentá-lo. As situações são distintas e as medidas de prevenção e enfrentamento devem ser criativas e articuladas.

As rodas de conversas possibilitam encontros dialógicos, criando possibilidades de produção e ressignificação de sentido saberes - sobre as experiências dos partícipes. Sua escolha baseia-se na horizontalização das relações de poder. Os sujeitos que as compõem se implicam, dialeticamente, como atores históricos e sociais críticos e reflexivos diante da realidade. Portanto, na roda, a fala é compreendida como expressão de modos de vida. As rodas são mais do que disposição física (circular) dos participantes e bem mais que uma relação custo-benefício para o trabalho com grupos. Elas são uma postura ético-política em relação à produção do conhecimento e à transformação social, efetivando-se a partir das negociaçóes entre sujeitos. $\mathrm{O}$ espaço da roda de conversa intenciona a construção de novas possibilidades que se abrem ao pensar, num movimento contínuo de perceber - refletir - agir - modificar, em que os participantes podem se reconhecer como condutores de sua ação e da sua própria possibilidade de "ser mais".

Os ACS e as enfermeiras das unidades menos violentas colocam que na comunidade foi formado um grupo de mulheres que discutem diversos temas, dentre eles violência contra a mulher, e realizam encontros mensais. Durante os encontros as mulheres colocam sobre seus anseios, medos e situações particulares. Na oportunidade dos encontros os profissionais colocam a temática de violência contra mulher sobre a rede de enfrentamento e solicitam sugestôes de temas para outros encontros.

No Programa Saúde da Família (PSF) as equipes encontram-se em posição estrategicamente favorável para intervir nas relações interpessoais familiares. A abordagem da família e o fortalecimento dos laços entre os profissionais de saúde e a população atendida constituem importantes estratégias que podem contribuir para a prevenção, a identificação e a intervenção em situações de violência familiar, permitindo aprofundar as relações, viabilizando a orientação dos conflitos para formas de enfrentamento menos destrutivas, mais saudáveis. Nessa perspectiva, os profissionais de saúde da família tornam-se atores da maior significância para o desenvolvimento das ações de prevenção da violência.

É importante ressaltar que mesmo na perspectiva do PSF nas ações de prevenção, ainda predomina uma lógica de atendimento sintomatológico voltada para o tratamento de lesões físicas e das sequelas dos atos violentos. Também a existência de problemas estruturais e conjunturais do setor saúde acaba por afetar a atuação dos profissionais. Nesse sentido, destacam-se: a sobrecarga dos profissionais de saúde; a inadequação dos espaços físicos das unidades para atendimento às vítimas; $\mathrm{e} o$ insulamento do setor frente às dificuldades de articulação para abordagem intersetorial do problema $^{11}$. Destacam-se também questóes relacionadas ao medo de represálias, falta de tempo e sentimento de impotência dos profissionais que contribuem para a atuação superficial, fragmentada e pouco resolutiva ${ }^{12}$.

Os profissionais da saúde da família apresentam grande potencialidade para a detecção, abordagem e acompanhamento qualificado das vítimas, pois esses lidam cotidianamente com situações de violência doméstica e sexual e vivenciam na prática as dificuldades inerentes ao processo de assistência e acompanhamento dos casos ${ }^{13}$.

O desconhecimento, por parte dos profissionais, da existência de redes de atenção às vítimas de violência foi também observado por Moreira e colaboradores ${ }^{14}$, que destacam a possibilidade de a desinformação acarretar prejuízos no apoio multiprofissional e intersetorial às vítimas. Essa posição é corroborada por Santos e Vieira ${ }^{15}$, ao afirmarem que o desconhecimento das redes de serviços existentes constitui fator de grande limitação para a abordagem integral dos problemas de saúde.

Evidenciou-se também no discurso dos profissionais que os encaminhamentos seriam uma forma de se livrar do problema, por não saberem lidar ou não quererem se envolver na situação. Conforme impressão na fala da Enfa/USF ${ }^{1}$ ela geralmente encaminha para se cobrir e registrar em prontuário, mas muitas vezes não tem a contra referência do caso, como ocorre nas fichas de notificação.

A falta do feedback dos casos referenciados não garante o fluxo e articulação bem definida. Assim o cuidado fica fragmentado, favorecendo o ciclo de violência. Por parte de toda equipe no que diz respeito à assistência nas unidades, a visita dos ACS, ao contrário disso, representaria apenas uma 'transferência' de responsabilidade a setores e profissionais que estariam mais bem preparados para esse tipo de atendimento.

Borsoi, Brandão e Cavalcanti ${ }^{16}$ observam que a identificação de um caso de violência doméstica deve ser apenas o início de um processo que busque apoiar as vítimas na superação do problema. Nesse contexto, advertem que os encaminhamentos não devem ser numa referência para fora do serviço e posterior perda de controle sobre os desdobramentos de sua ação, mas uma estratégia destinada a envolver diversos segmentos na articulação para a resolução do problema. Diante da perspectiva da integralidade do cuidado, torna-se fundamental que os encaminhamentos se façam de maneira articulada com o setor de destino e que o vínculo do usuário seja mantido em ambos os serviços, de maneira que se fortaleçam os pontos de encontro 
e a articulação que devem existir na rede.

Ainda há uma lacuna de conhecimentos em relação à abordagem e tratamento do agressor ${ }^{17}$. Conforme destacam Lima, Büchele e $\mathrm{Clímaco}^{18}$, essa questão não se resume à escassez de publicações, mas sobretudo à diminuta presença de serviços que prestam atendimento e acompanhamento aos homens autores da violência. A abordagem do agressor para além da repressão e punição não se configura como política pública no Brasil e países latino-americanos. Assim, o envolvimento dos homens na prevenção, atenção e enfrentamento à violência doméstica permanece incipiente, o que pode denotar limitações na abordagem do problema.

Em uma perspectiva de articulação em rede, de forma interdisciplinar e intersetorial, deve-se envolver assim amplos setores da sociedade, como Conselhos de Direitos, Delegacia de Mulheres, Organização não governamental (ONGs), hospitais, unidades de saúde, escolas, centros de referência das mulheres, movimentos de mulheres, entre outros. Esses movimentos de resistência têm caráter de denúncia, mas também de reivindicação, implicando a emergência de novos sujeitos sociais e cenários políticos, caracterizados pela transversalidade na luta por demandas de direitos.

Assim, evidencia-se a necessidade de investimentos em educação permanente como estratégia de sensibilização e qualificação dos profissionais da estratégia de saúde da família para o enfrentamento da violência contra a mulher. A educação contínua, a partir da prática reflexiva e da socialização/integração dos diversos saberes, contribui para melhorar o desempenho profissional, desenvolver novas competências e construir novos conhecimentos. Propõe-se a formar atores crítico-reflexivos que avaliam e modificam sua prática e estão em constante busca de novos saberes ${ }^{19}$. No entanto, a educação permanente só se operacionaliza quando ocorre a mudança institucional das práticas de educação e formação profissional ${ }^{19}$. Para Santos e Santos ${ }^{20}$, o desenvolvimento de ações de Educação em Saúde fundamentadas nas tendências pedagógicas libertadoras tem demonstrado resultados importantes para a transformação da práxis. Nesse sentido, há necessidade de superar as ações de treinamentos esporádicos, que geram modificaçôes localizadas, individuais e temporárias, com natural incapacidade de provocar transformações profundas sobre

\section{Conforme esse}

programa de

mulheres de paz, as

vítimas da violência

passam a receber uma

bolsa do governo

federal e realizam

ações de prevenção à

violência.

o agir dos profissionais.

Através da pesquisa de campo e das impressões dos relatos de profissionais e gestores no enfrentamento da violência contra mulheres e baseada na literatura, entre os desafios expostos está a materialização dos pressupostos de trabalho em rede, como a horizontalidade e o enfrentamento das relações de poder existentes entre diferentes políticas e setores. Como possibilidades destacam-se a articulação de profissionais de diferentes áreas de saber, no atendimento à complexidade das demandas trazidas por mulheres em situação de violência contra a mulher.

Vale destacar aqui ainda que alguns programas têm sido desenvolvidos no âmbito de outras políticas setoriais no sentido da prevenção à violência contra a mulher. Exemplo disso é o Programa Mulheres da Paz que beneficia mulheres que pertençam às redes de parentesco e redes sociais dos jovens, foco territorial do Programa Nacional de Segurança Pública com Cidadania (PRONASCI), desenvolvido pelo Ministério da Justiça a partir de 2007. O programa marca uma iniciativa inédita no enfrentamento à criminalidade no país, articula políticas de segurança com ações sociais; prioriza a prevenção e busca atingir as causas que levam à violência, sem abrir mão das estratégias de ordenamento social e segurança pública ${ }^{21}$.

Conforme esse programa de mulheres de paz, as vítimas da violência passam a receber uma bolsa do governo federal e realizam ações de prevenção à violência. O acompanhamento e o monitoramento do programa visa garantir a efetividade e a transparência na implementação do $\mathrm{Mu}$ lheres da Paz, assegurando que os benefícios efetivamente sejam repassados às mulheres.

Segundo Grossi e colaboradores ${ }^{22}$, outra iniciativa do PRONASCI foi capacitar as mulheres para o mercado de trabalho e formas de geração de renda. Um grupo de mulheres formou uma cooperativa (malharia) e confecciona os uniformes para a rede de ensino. Outro grupo passou a produzir bijuterias para comercialização nas feiras do município e também houve capacitação para as mulheres rurais através de convênios com a Empresa de Assistência Técnica e Extensão Rural (EMATER).

\section{CONCLUSÃO}

O estudo revelou que os profissionais não sabem lidar diante da situação de violência, não conhecem os fluxos para encaminhamento e apontam para a necessidade de maior divulgação desses a fim de garantir à mulher o atendimento às suas necessidades. Conforme a pesquisa, a falta de capacitação é a realidade dos profissionais de saúde que não se encontram preparados para oferecer uma atenção que tenha impacto efetivo à saúde das vítimas. Para isso, faz-se necessário o suporte dos diversos serviços de atenção: jurídico, policial, social, de geração e renda, de habitação e de saúde. Faz-se necessário, também, empreender estudos de construção de saberes e práticas dos prestadores interdisciplinar, para que atendam nos diversos espaços de atuação 


\section{artigo}

Gomes Silva, M.; Estrela, F.M.; Peixoto, T.M.; Moreira, R.M.O.; Morais, A.C.; Couto, P.L.S.; Portela, P.L.S.

Violência contra a mulher em Feira De Santana-BA: Encaminhamentos e articulação em rede

suprindo todas as lacunas das vítimas no âmbito jurídico, psicológico e de saúde.

Acredita-se que os resultados desse es- tudo possam contribuir para maior visibilidade das questões que entravam o processo de atenção à mulher em situação de violência doméstica, oferecendo subsídios para se pensar ações e estratégias de prevenção e enfrentamento do fenômeno.

\section{REFERÊNCIAS}

1. Fórum Brasileiro de Segurança Pública. Violência doméstica durante a pandemia de Covid-19. Ed 3. 2020 [citado 2021 fev 11]. Disponivel em: https://forumseguranca.org.br/wp-content/uploads/2018/05/violencia-domestica-covid-19-ed03-v2.pdf

2. Instituto de Pesquisa Econômica Aplicada. Fórum Brasileiro de Segurança Pública. Atlas da violência: 2017. Rio de Janeiro: IPEA, FBSP, 2017 [acesso 2019 mar 25]. Disponivel em: https:/assets-dossies-ipg-v2.nyc3.digitaloceanspaces.com/sites/3/2018/04/IPEA_ FBSP_atlasdaviolencia2017.pdf

3. Valesca C, Caesar G, Reis T. Mesmo com queda recorde de mortes de mulheres, Brasil tem alta no número de feminicídios em 2019. Rio de Janeiro: G1, 2020 [citado 2021 fev 11]. Disponivel em: https:// g1.globo.com/monitor-da-violencia/noticia/2020/03/05/mesmocom-queda-recorde-de-mortes-de-mulheres-brasil-tem-altano-numero-de-feminicidios-em-2019.ghtml

4. Organização Mundial da Saúde. Informe mundial sobre la violencia y salud. Genebra: OMS, 2002.

5. Bandeira L, Almeida TMC. Desafios das políticas e ações em saúde diante da violência contra as mulheres. SER Social, 2008; 10(22): 183-212.

6. Silva RA, Araújo TVB, Valongueiro S, Ludermir AB. Enfrentamento da violência infligida pelo parceiro íntimo por mulheres em área urbana da região Nordeste do Brasil. Rev. Saúde Pública [Internet]. 2012 [citado 2021 Jan 29]; 46(6): 1014-1022. Disponivel em: http://www.scielo.br/scielo.php?script=sci_arttext\&pid=S0034-89102012000600011\&Ing=pt. Epub 01-Fev-2013. https://doi.org/10.1590/S0034-89102013005000007.

7. Triviños ANS. Introdução à pesquisa em ciências sociais: a pesquisa qualitativa em educação. São Paulo: Atlas, 2006.

8. Minayo MCS. O desafio do conhecimento. 11. ed. São Paulo: Hucitec, 2008

9. Bahia. Secretaria de Segurança Pública. Polícia alcança maior redução de CVLIs dos últimos 6 anos [Internet]. Salvador: Secretaria de Segurança Pública, 2018 [acesso 2019 mar. 29]. Disponível em: http://www.ssp.ba.gov.br/2018/12/4932/Policia-Baiana-alcancamaior-reducao-de-CVLIs-dos-ultimos-6-anos.html

10. Rodríguez-Bolaños RA, Márquez-Serrano M, Kageyama-Escobar ML. Violencia de género: actitud y conocimiento del personal de salud de Nicaragua. Salud Publica Mexico, 2005; 47(2): 134-44.

11. Ferrante FG, Santos MA, Vieira EM. Violência contra a mulher: percepção dos médicos das unidades básicas de saúde da cidade de Ribeirão Preto, São Paulo. Interface, 2009; 13(31): 287-99.

12. Wanderbroocke ACNS, Moré CLO. Estrutura e funcionamento familiar e a violência contra idosos. Psicologia Argumento. [Internet]. 2013 [acesso 2019 mar 26]; 31(74): 147- 268. Disponível em: http://www2.pucpr.br/reol/pb/index.php/pa?dd1=12231\&dd99=view\&dd98=pb
13. Andrade EM, Nakamura E, Paula CS, Nascimento R, Bordin IA., Martin D. A visão dos profissionais de saúde em relação à violência doméstica contra crianças e adolescentes: um estudo qualitativo. Saude soc. [Internet]. 2011 [cited 2021 Jan 29]; 20(1): 147-155. Available from: http://www.scielo.br/scielo.php?script=sci_arttext\&pid=S0104-12902011000100017\&lng=en. https://doi. org/10.1590/S0104-12902011000100017.

14. Moreira SNT, Galvão LLLF, Melo COM, Azevedo GD. Violência física contra a mulher na perspectiva de profissionais de saúde. Rev. Saúde Pública [Internet]. 2008 [cited 2021 Jan 29]; 42(6): 10531059. Available from: http://www.scielo.br/scielo.php?script=sci arttext\&pid=S0034-89102008000600011\&Ing=en. Epub Oct 03, 2008. http://dx.doi.org/10.1590/S0034-89102008005000058.

15. Santos MA, Vieira EM. Recursos sociais para apoio às mulheres em situação de violência em Ribeirão Preto, SP, na perspectiva de informantes-chave. Interface (Botucatu) [Internet]. 2011 [cited 2021 Jan 29]; 15(36): 93-108. Available from: http://www.scielo.br/scielo. php?script=sci_arttext\&pid=S1414-328f32011000100008\&lng=en. https:/doi.org/10.1590/S1414-32832011000100008.

16. Borsoi TS, Brandão ER, Cavalcanti MLT. Ações para o enfrentamento da violência contra a mulher em duas unidades de atenção primária à saúde no município do Rio de Janeiro. Interface. 2009; 13(28): 165-174.

17. Gontijo DT, Alves HC, Paiva MHP, Guerra RMR, Kappel VB. Violência e saúde: uma análise da produção científica publicada em periódicos nacionais entre 2003 e 2007. Physis [Internet]. 2010 [cited 2021 Jan 29]; 20(3): 1017-1054. Available from: http://www.scielo.br/scielo.php?script=sci_arttext\&pid=S0103-73312010000300017\&Ing=en

18. Lima DC, Büchele F, Clímaco D. Homens, gênero e violência contra a mulher. Saúde e Sociedade [Internet], 2008 [acesso 2019 mar 26]; 17(22): 69-81. Disponivel em: http://www.scielo.br/pdf/sausoc/v17n2/08.pdf

19. Brasil. Ministério da Saúde. Secretaria de Gestão do Trabalho e da Educação na Saúde. Política Nacional de Educação Permanente em Saúde. Brasília: Ministério da Saúde, 2009b.

20. Santos IMV, Santos AM. Acolhimento no Programa Saúde da Familia: revisão das abordagens em periódicos brasileiros. Revista de Salud Publica, 2011; 13(4): 703-16.

21. Sorj B, Gomes C. O gênero da "nova cidadania": o Programa Mulheres da Paz. Sociologia \& Antropologia [Internet]. 2011 [acesso 2015 jun 26]; 1(2):147-164. Disponivel em: http:/www.scielo.br/scielo.php?script=sci_arttext\&pid=S2238-38752011000200147\&lng=en\&nrm=iso

22. Grossi PK, Pedersen JR, Vincensi JG, Almeida SM. Prevenção da violência contra mulheres: desafios na articulação de uma rede intersetorial. Athenea Digital [Internet]. 2018 [acesso 2018 set. 30]; 12(3): 267-277. Disponivel em: https://atheneadigital.net/article/ view/v12-n3-krieger-pedersen-vincensi-etal 